\title{
Analysis of the influences of bracket and force system in different directions on the moment to force ratio by finite element method
}

\author{
Yu Yang and Wencheng Tang ${ }^{*}$
}

\begin{abstract}
The human teeth face a variety of ways to move during orthodontic treatment. The types of orthodontic tooth movements have linked to the moment to force $(M / F)$ ratio. This paper aims to determine the precise M/F ratios that generate different types of movements for the models with and without bracket, and the M/F ratios are compared between the force systems applied in buccal-lingual and distal-mesial direction for the mandible canine by using finite element method (FEM). A segment of mandible canine obtained from autopsy had been scanned with microcomputed tomography, and two finite element models with and without brackets were established. Each canine model was subjected to a force of $100 \mathrm{cN}$ and varied M/F ratios from $0 \sim 12$ in buccal-lingual and distal-mesial directions. We find that the model with a bracket required larger M/F ratio compared to the model without a bracket for the same tooth movement. For the different directions, the value of M/F ratio is larger in the buccal-lingual than in the distal-mesial direction. The geometry of the tooth and the PDL are gained from a regular patient. Therefore, the results are applicable to any canine, and the precise M/F values provide a theoretical basis for the orthodontist design optimal force system.
\end{abstract}

Keywords: M/F ratios, Tooth movement, Bracket, Finite element analysis

\section{Introduction}

Tooth movement occurs due to the changes in the strain-stress distribution within the periodontal ligament (PDL) after the application of a force system to the bracket. The types of tooth movements can be summarized as follows: tipping, translation, rotation, and root movement. In addition, tipping can be further classified into uncontrolled tipping and controlled tipping. As an important parameter, the moment to force $(\mathrm{M} / \mathrm{F})$ ratio plays a key role in determination of the type of tooth movement $[1,2]$.

According to the classic theory, Burstone et al. [3] studied the localization of the center of resistance (CRe) at an approximate of $40 \%$ from the apex to the measured length between the alveolar crest and the tooth root based on a mathematical model. Moreover, the center of

\footnotetext{
* Correspondence: tangwc@seu.edu.cn

School of Mechanical Engineering, Southeast University, Nanjing, People's Republic of China
}

rotation (CRot) can be calculated by the Burstone's theoretical formula $\left(y=0.068 h^{2}(\mathrm{~F} / \mathrm{M})\right)$, where $h$ is the distance between apex and the alveolar crest and $y$ denotes the distance from the CRe to the CRot. Provatidis CG [4] validated the formula through the finite element method (FEM). Christiansen [5] confirmed that the CRot is related to the $\mathrm{M} / \mathrm{F}$ ratio by application of different force levels on maxillar central incisors, but the CRot seemed to be positioned more inclined to apical direction by FEM compared to mathematical calculation. These results are confirmed by many clinical cases. The different locations of CRe between theoretically calculated and experimental results can be attributed mainly to the limitation of the model, 2D model established and the assumption of linear behavior for the PDL using FEM.

In recent years, numeric methods have been widely used to calculate the stress/strain in the PDL, and FEM has been the choice of orthodontic researchers 
frequently. Some previous literatures [6, 7] confirmed the limitations on the finite element (FE) results due to the complexity of shape and composition of the periodontal tissue. Many attempts on the relationship between the force system and tooth movement have been made as well as the reaction of the surrounding tissues have been made; however, it is still an open question. Cattaneo et al. [8] simulated the pure translation of the tooth with an M/F ratio of 10 and 12 respectively for premolar and canine. Athicha et al. [9] suggested that bodily movement of incisor happened when the ratio is 11.65 at zero inclination. Zhipeng Liao et al. [10] proposed that an $\mathrm{M} / \mathrm{F}$ ratio of approximately $10.2,9.7$, and 8.8 can generate the bodily movements of maxilla canine, incisor, and first premolar. Cai et al. [11] attempted to find appropriate $\mathrm{M} / \mathrm{F}$ ratio for translation and controlled tipping movement. However, they neglected the influence of a bracket and the direction of external force on the $\mathrm{M} / \mathrm{F}$ ratio. In quantitative analysis, the influences can help the orthodontist design ideal orthodontic force system for different patients.

Application of FEM fitted to a dental biomechanic commenced in the mid 1970s. This technology has particularly attracted a number of orthodontic investigators since they can establish an elaborate 3D model, simulate tooth movement accurately when subjected to various orthodontic forces, and provide an excellent visualization profile of the displacement and stress fields that occur in the PDL and surrounding tissues. In finite element (FE) analysis, each material component is subdivided into a large number of elements. An accurate tooth model including the physical properties and geometry of the component is required for calculation. As an important connective soft tissue, the PDL transmits orthodontic force from the tooth to the alveolar bone, resulting in tooth movement. Even though the PDL is known to represent a nonlinear viscoelastic behavior, many literatures [12-15] assumed a linear elastic behavior for the PDL in FE analysis. The hyperelastic, viscoelastic, and biphasic poroelastic behaviors are also adopted to descript the mechanical properties of the PDL under different loading conditions [16-19]. The PDL's nonlinear viscoelastic behavior can be considered as an instantaneous elastic and time-dependent nonlinear viscous behavior, while a hyperelastic model [17] can describe the short-term instantaneous behavior.

Orthodontic treatment is a long-term process with repeated occurrences and even side effects (if you do not expect to have tooth movement). The reason for this is that the orthodontists can not accurately control the orthodontic force. Studying orthodontics and the way the tooth moves can help orthodontists to develop a more rational orthopedic plan and design to ultimately improve orthodontic treatment efficiency. Previous studies have focused on teeth movements when a force or moment is applied to the tooth directly, the effect of bracket, and the direction of the force on the M/F ratios based on suitable material assumption for the PDL which are rarely performed in orthodontics. The aim of this study is to determine the precise $\mathrm{M} / \mathrm{F}$ ratio that can generate uncontrolled tipping, controlled tipping, and translation movements for the mandible canine models with and without brackets, as well as the force systems applied in buccal-lingual and distal-mesial directions.

\section{Material and methods}

To achieve more precise model, the mandible canine model has been constructed based on CT images from a 16-year-old female patient with a resolution of approximately $0.25 \mathrm{~mm} /$ pixel. First, 3D outline shapes of the alveolar bone and tooth, which consist of cloud points, are established using an image-processing software (Mimics 10.1). Afterward, the point cloud data are imported to the CATIA P3 V5R21 software to create the solid model. Finally, all completed solid models were imported in ABAQUS 6.1 for finite element analysis. The model is composed of a tooth, PDL, and a section of the alveolar bone. The canine length is $25.6 \mathrm{~mm}$ of which the root length is $13.5 \mathrm{~mm}$, with a distal-mesial diameter of $7.0 \mathrm{~mm}$ and a buccal-lingual diameter of $7.9 \mathrm{~mm}$. To simplify, the PDL tissue is modeled with an average thickness of $0.2 \mathrm{~mm}$ between the root of the tooth and the alveolar bone. For the FE models, the tooth and alveolar bone are meshed by an eight-node tetrahedral (C3D8) element and the PDL is meshed by an eight-node tetrahedra $(\mathrm{C} 3 \mathrm{D} 8 \mathrm{H})$ element; the bracket is meshed by an eight-node hexahedra (C3D8) element. A mesh convergence study for each component is completed in accordance with that reported in literature [20], and the results are shown in Table 1 . This produced approximately $1,431,400$ nodes with $1,135,200$ elements.

As mentioned earlier, the alveolar bone deformation is caused by the stresses/strains in PDL. In reality, the PDL is likely to react after the force was exerted to the bracket and transmit the force to the alveolar bone, resulting in bone resorption and apposition [21]. Hence, the tooth movement is associated with the material properties of the PDL. The model contains four materials: the bone, dentin, PDL, and stainless steel. Elastic modulus and Poisson's ratio as the parameters of material properties have assigned to the model component in the FE software. Due to the elastic modulus of the tooth and bone are 1000-3000 times of the PDL, the variation

Table 1 The results of mesh convergence

\begin{tabular}{lllll}
\hline Components & Tooth & Alveolar bone & Bracket & PDL \\
\hline Element size & $0.2 \mathrm{~mm}$ & $0.2 \mathrm{~mm}$ & $0.2 \mathrm{~mm}$ & $0.1 \mathrm{~mm}$ \\
\hline
\end{tabular}


of material property settings for them have a minimal effect on the FE result of the PDL. All the materials except the PDL are assumed to be linear elastic and homogeneous for the analyses as acceptability in existing literature [21]. The parameter of material properties are obtained from published data $[22,23]$ as list in Table 2. Due to the PDL is considered as a nonlinear material, a nonlinear stress-strain curve from Wei ZG et al. [24] has adopted to fit by a hyperelastic model, namely a second-order Ogden model is given as follows.

$$
U=\sum_{i=1}^{2} \frac{2 \mu_{i}}{a_{i}^{2}}\left(\bar{\lambda}_{1}^{a_{1}}+\bar{\lambda}_{2}^{a_{1}}+\bar{\lambda}_{3}^{a_{1}}\right)+\sum_{i=1}^{2} \frac{1}{D_{i}}\left(J^{e l}-1\right)^{2}
$$

where $U$ is the strain energy per unit of reference volume, $a_{i}$ and $\mu_{i}(i=2)$ are the material parameters. $\bar{\lambda}=J^{-1 / 3} \lambda_{i}$ are the deviatoric principal stretches. These parameters are obtained (Table 3) through Abaqus material evaluator.

Different $\mathrm{M} / \mathrm{F}$ ratios are applied to the model to simulate orthodontic tooth movements. In accordance with previous literature, a force of $100 \mathrm{cN}$ on the model with bracket and without bracket acted in the buccal-lingual direction for the canine [25]. The chosen value is corresponded to the force level used in clinical practice [5]. The force is kept constant while the M/F ratios vary from 0 (uncontrolled tipping) to 12 (pure translation). The same force system has applied to the model in distal-mesial direction (Fig. 1). The interface between the tooth and the PDL, alveolar bone and the PDL as well as between tooth and bracket are defined as "tie" connection. For the boundary conditions, the constraint in all directions is applied for the nodes located at the base of the alveolar bone. Several typical tooth movements and the location of CRot have been performed. For each analysis, the displacements of the crown and apex are calculated.

\section{Results}

For the model with bracket, the results that varied M/F ratios resulting in different tooth movements are shown in Fig. 2 (the shaded represents undeformed shape and substance represents deformed shape). It can be seen that uncontrolled tipping is obtained when the M/F ratio is 0 . With the increased of $\mathrm{M} / \mathrm{F}$ ratio, the location of CRot moves gradually to the apex of the root until produced controlled tipping. When an M/F ratio of 10.6 is

Table 2 Mechanical property of four materials

\begin{tabular}{lllll}
\hline Material & Tooth & PDL & Alveolar bone & Bracket \\
\hline Elastic modulus (MPa) & 18,600 & Hyperelastic & 13,700 & $2.1 \times 10^{5}$ \\
Poisson's ratio & 0.3 & - & 0.3 & 0.3 \\
\hline
\end{tabular}

Table 3 Parameters of the Ogden model

\begin{tabular}{lllll}
\hline Parameters & $\mathrm{alO}_{1}(\mathrm{MPa})$ & $a_{2}(\mathrm{MPa})$ & $\mu_{1}(\mathrm{MPa})$ & $\mu_{2}(\mathrm{MPa})$ \\
\hline Value & 19.20265 & -4.8886 & 0.01107 & 0.03175 \\
\hline
\end{tabular}

applied to the bracket, the CRot is situated infinitely far from the root of canine. The canine's pure translation can be observed; we can see a relative large deformation in central of the tooth and a minor deformation in the zone of cusp and root. Similar change trends have appeared for another FE simulation (Fig. 3).

Apart from the position of the CRot, the displacements of the crown and apex are also important basis to determine the types of the tooth movements. Figure 4 displays the results of the displacements of the crown and apex for the models with and without bracket in buccal-lingual direction when the varied M/F ratios have applied to the model (positive represents lingual direction and negative represents buccal direction). During uncontrolled tipping $(\mathrm{M} / \mathrm{F}=0)$, the crown of the canine with and without bracket are displaced 0.0114 and $0.0152 \mathrm{~cm}$ buccally, respectively, whereas the apex of canines are moved 0.0025 and $0.0033 \mathrm{~cm}$ lingually for the models with and without bracket, respectively. With the $\mathrm{M} / \mathrm{F}$ ratio increasing, both the displacements of the crown and apex gradually decreases until the movement of the apex tended to zero, and then the controlled tipping occurred, where the values of the M/F ratio now are 6.4 and 7.3 for the models with and without bracket, respectively. As further increasing the $\mathrm{M} / \mathrm{F}$ ratio, the apex started moving in opposite directions. When the crown and apex move the same distance along the force direction, pure translation occurred for the two models. For the model with bracket, both crown and apex moved lingually $0.000078 \mathrm{~cm}$ when the $\mathrm{M} / \mathrm{F}$ ratio is 10.6 . Meanwhile, for the model without bracket, both crown and apex move lingually $0.000112 \mathrm{~cm}$ when the M/F ratio is 11.5. After that, the movement of the crown tends to be zero, and the canine exhibits root movement.

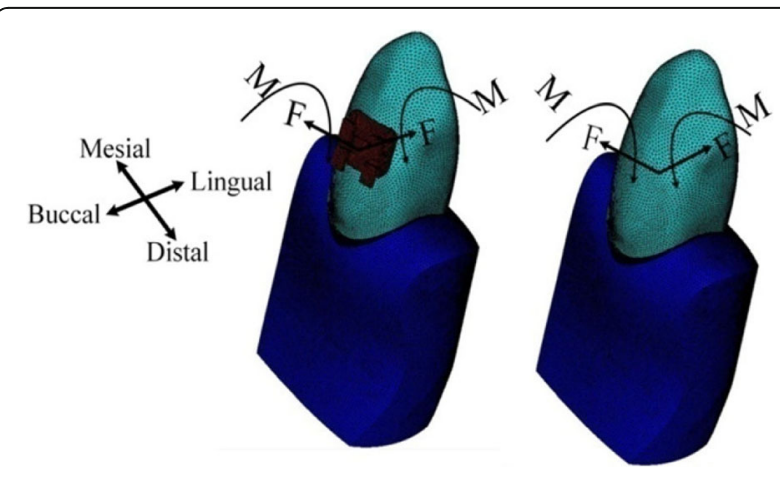

Fig. 1 A constant force and varied moments applied to the models with bracket and without bracket 

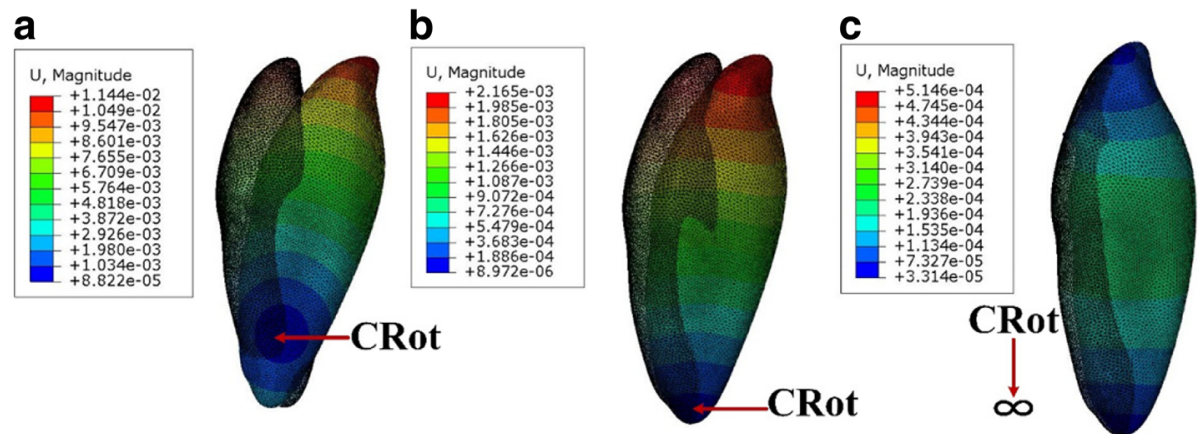

Fig. 2 Several types of tooth deformation enlarged by a factor of 500, the force systems applied in buccal-lingual direction. a Uncontrolled tipping $(M / F=0)$. $\mathbf{b}$ Controlled tipping $(M / F=6.4)$. $\mathbf{c}$ Pure translation $(M / F=10.6)$

The same force systems are applied to the model in distal-mesial direction. Figure 5 displayed a similar tendency compared to buccal-lingual direction (positive represents mesial direction and negative represents distal direction), but there are some differences in displacements and M/F ratios. For the model without bracket, the crown and apex of the canine are displaced $0.00620 \mathrm{~cm}$ mesially and $0.00159 \mathrm{~cm}$ distally during

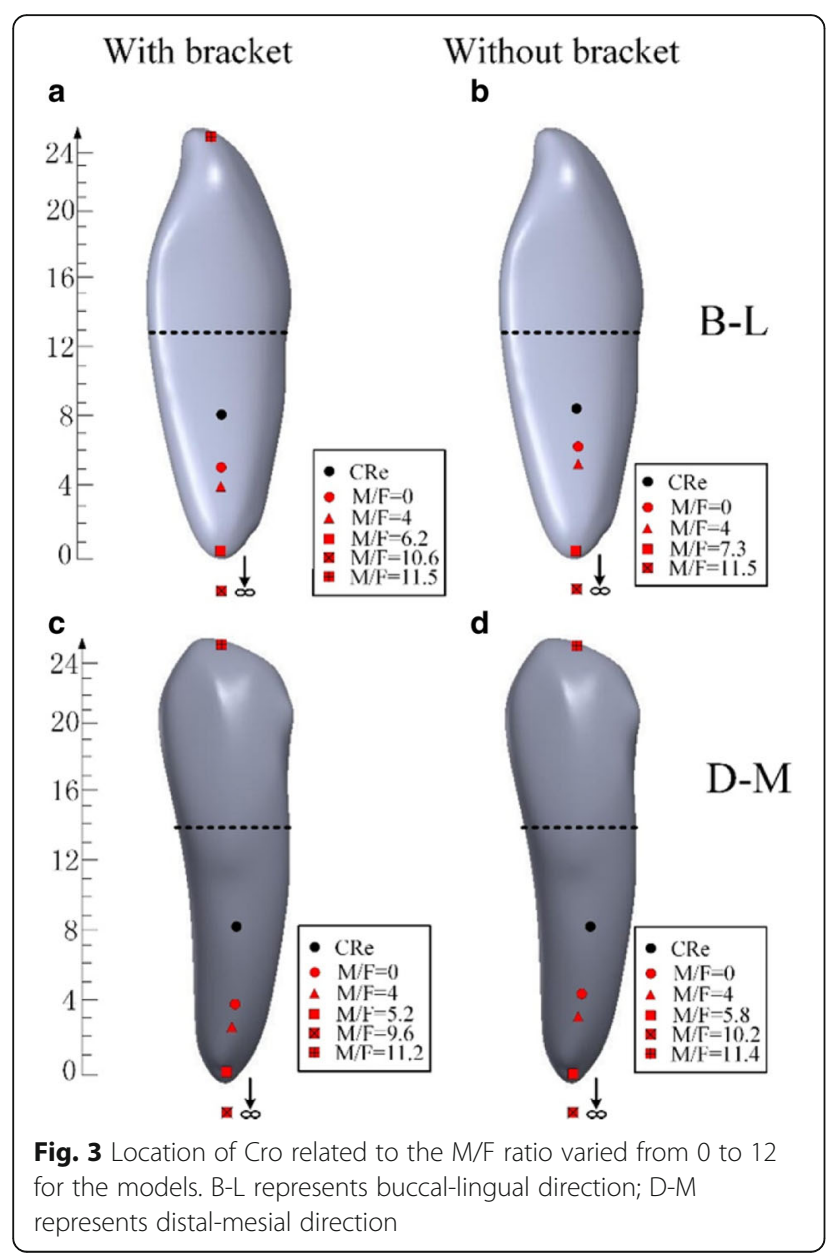

uncontrolled tipping movement $(\mathrm{M} / \mathrm{F}=0)$. With the $\mathrm{M} /$ F ratios increase to 5.8 and 10.2, controlled tipping and pure translation occurred, the crown is displaced $0.00185 \mathrm{~cm}$ mesially (apex was nearly zero), and the crown and apex are displaced $0.00074 \mathrm{~cm}$. On the other hand, for the model with bracket, the displacements of the crown and apex are $0.00221 \mathrm{~cm}$ mesially and $0.000484 \mathrm{~cm}$ distally during uncontrolled tipping $(\mathrm{M} / \mathrm{F}=$ 0 ). Controlled tipping can occurred when the M/F ratio is 5.2 , and an $\mathrm{M} / \mathrm{F}$ ratio of approximately 9.6 generates the translational movement.

The M/F ratios in buccal-lingual and distal-mesial directions for the two models are summarized in Table 4. For generating a same tooth movement, more M/F ratios are always needed for the model without bracket compared to the model with bracket. Furthermore, more $\mathrm{M} / \mathrm{F}$ ratios are also required in buccal-lingual direction than in distal-mesial direction when the same tooth moving is simulated.

The relationships between the maximum and minimum principal stresses in the PDL and the M/F ratio for the model with bracket have been plotted in Fig. 6. The locations of the maximum principal stresses for each $M$ / $F$ ratio and the ratio produced from the different types of canine movements are also shown (Fig. 6). It has been found that all values of maximum principal stresses are positive and the values are negative for minimum principal stresses, so they can be called as maximum tensile stress and maximum compressive stress, respectively. The maximum tensile and compressive stress occurred at midroot on buccal and lingual sides, respectively. When the M/F ratio is low (0-5.6), both the maximum tensile and compressive stress concentrate on apex. With the M/F ratio increased (6-12), the locations of maximum tensile and compressive stress move from the cervical area to the midroot area on buccal and lingual sides. Figure 7 shows the stress distribution of maximum and minimum principal stresses for the PDL during the uncontrolled tipping $(\mathrm{M} / \mathrm{F}=0)$, It is found that there is 


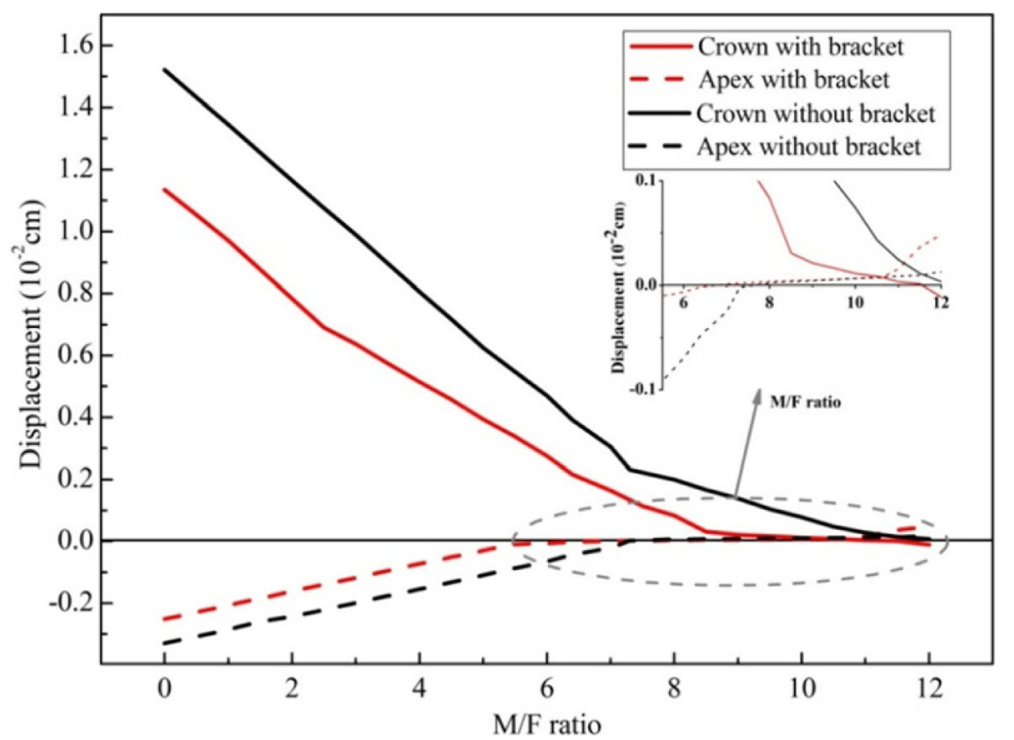

Fig. 4 Buccal-lingual displacements of the crowns and apices of the mandible canine, a force of $100 \mathrm{cN}$ and the values of $\mathrm{M} / \mathrm{F}$ ratio is increased from 1 to 12

no significant difference for the stress distribution of the PDL between the two models.

Orthodontists usually apply orthodontic force and moment to the bracket. The orthodontic force system is mostly derived from arch wire, elastic rubber, bands, or various screws and so on. On the other hand, more and more people are inclined to use invisible appliance without bracket. The results of this study can lay a theoretical foundation for medical researchers to design more individualized orthodontic tools.

\section{Discussion}

The target of orthodontic treatment is to shift maloccluded teeth a predetermined distance in a prescribed direction. The accuracy of M/F ratios and the location of the $\mathrm{CRe}$ are the two key factors to obtain satisfaction treatment result. Many researchers reported that CRe is located at 24 to $50 \%$ of the distance from the alveolar crest to the apex based on mathematical calculation and FEM [26-28]. Nevertheless, in reality, clinicians preferred to employ M/F ratios for a specific type of tooth

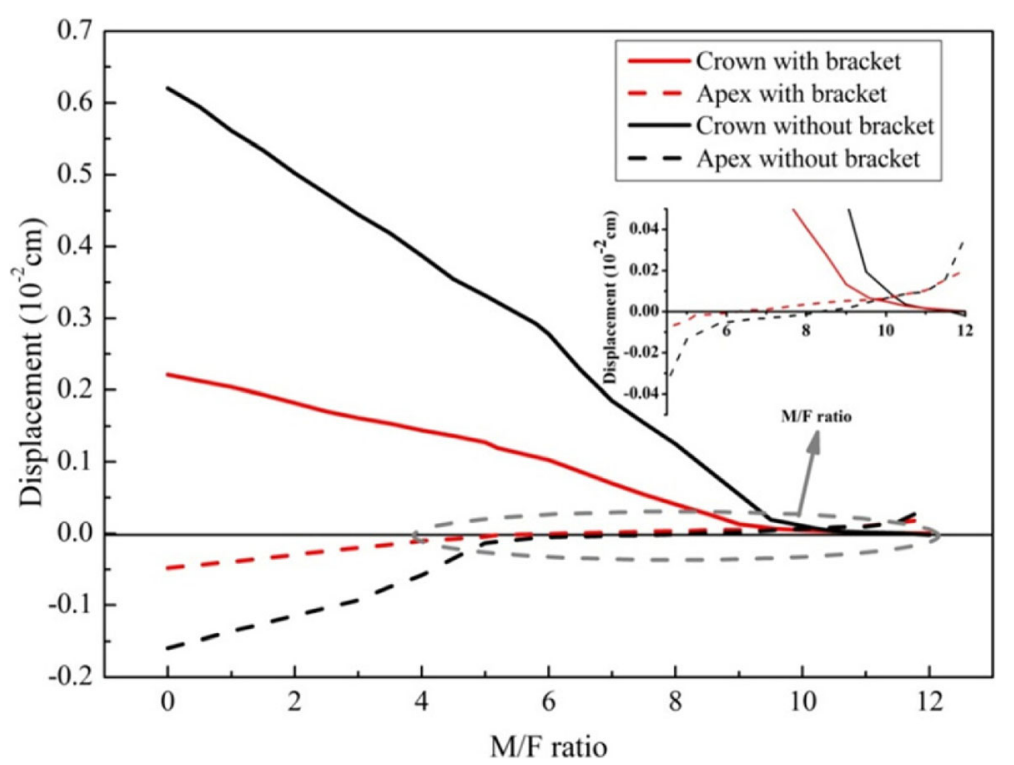

Fig. 5 Distal-mesial displacements of the crowns and apices of the mandible canine, a force of $100 \mathrm{cN}$ and the values of M/F ratio is increased from 1 to 12 
Table 4 The types of canine movements correspond to the M/F ratios

\begin{tabular}{|c|c|c|c|c|}
\hline & \multicolumn{4}{|l|}{ M/F ratio } \\
\hline & \multicolumn{2}{|c|}{ Buccal-lingual direction } & \multicolumn{2}{|c|}{ Distal-mesial direction } \\
\hline & With bracket & Without bracket & With bracket & Without bracket \\
\hline Uncontrolled tipping & $0 \sim 5.6$ & $0 \sim 6.8$ & $0 \sim 4.2$ & $0 \sim 4.9$ \\
\hline Controlled tipping & 6.2 & 7.3 & 5.2 & 5.8 \\
\hline Translational & 10.6 & 11.5 & 9.6 & 10.2 \\
\hline
\end{tabular}

movement because of clinical experience, without fully understanding the precise location of CRe for a tooth. It is difficult for the orthodontists to determine the exact location of CRe by mathematical deduction and FE calculation. However, the relationship between M/F ratios and the type of tooth movement remains inaccurate attributing to the difference form patient to patient, repeated visits to orthodontists for adjustment of orthodontic force system have to be necessitated.

The aim of this study is to determine the precise value of $\mathrm{M} / \mathrm{F}$ ratios that generate different types of tooth movements and compare the $\mathrm{M} / \mathrm{F}$ ratios between the models with bracket and without bracket, as well as different directions (buccal-lingual and distal-mesial directions). A patient-specific canine has chosen as the model to study. For the same type of tooth movement, the value of $\mathrm{M} / \mathrm{F}$ ratio is larger for the model without bracket than for the model with bracket. In additional, the displacement amplitude is lower for the model with bracket. This can be explained that the bracket model has larger area to sustain orthodontic force. By looking at the force system applied in different directions, one can notice that the displacements of the crown and the apex for the models in distal-mesial direction are far less than for the models in buccal-lingual direction. More $\mathrm{M} / \mathrm{F}$ ratios are needed for the models in buccal-lingual direction (Figs. 4 and 5). There are principal results of two causes, in which there is a smaller contact area of the canine at the lingual side compared to the mesial side (Fig. 3) and the force system did not pass though the CRe when the force system applied to the models in buccal-lingual direction.

A number of studies have been investigating the influence of different factors on the position of the CRot. An FEM conducted by Provatidis [4], concluded that the position of the CRot is linked not only to root length but also the root diameter, the material properties of the PDL, as well as its thickness. D. Vollmer et al. [23] compared the difference of position of CRot between an upper human canine and idealized tooth model. By applying a range of values of $\mathrm{M} / \mathrm{F}$ ratio, Cattaneo, $\mathrm{P}$. M [29] found that the position of the CRot of premolar and canine gradually approaches to the apex with an increase of $\mathrm{M} / \mathrm{F}$ value (before translational movement occurred) applied to the model, which is corresponding with this study. Fig. 3 displays the relationship between the position of CRot and the M/F ratio for the models with and without bracket when the force system applied in buccal-lingual and distal-mesial direction. The position of CRot is highest for the model without bracket, followed by the model with bracket, and is higher in buccal-lingual than in distal-mesial direction when equal force system is applied to the models (uncontrolled tipping).

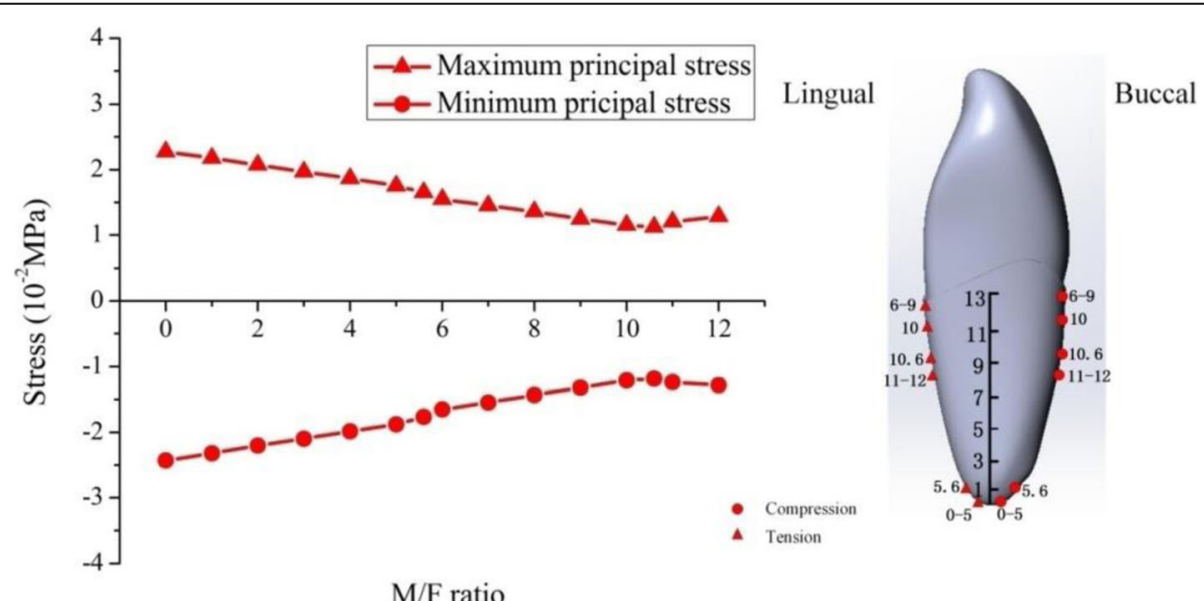

Fig. 6 Relationships between the M/F ratio(buccal-lingual direction) and principal stress for the model with bracket. Diagrams on the right show locations of maximum compressive and tensile stress 


\section{With bracket}
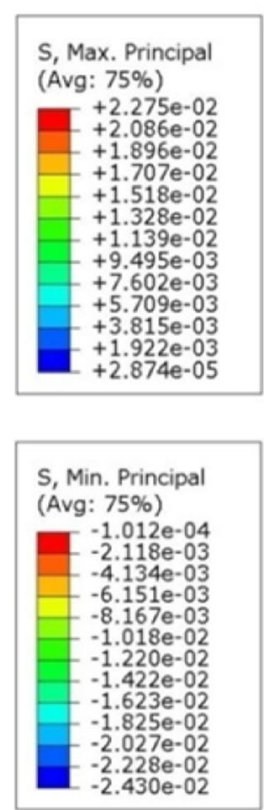
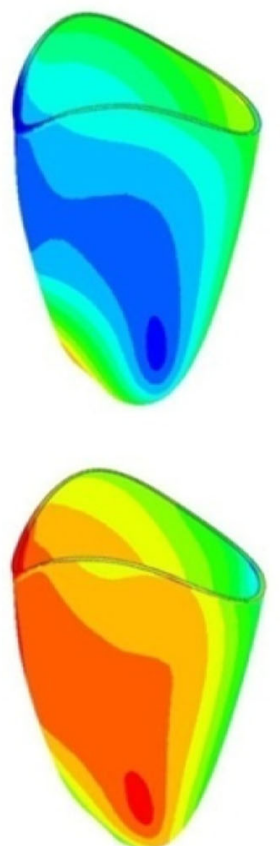

Without bracket
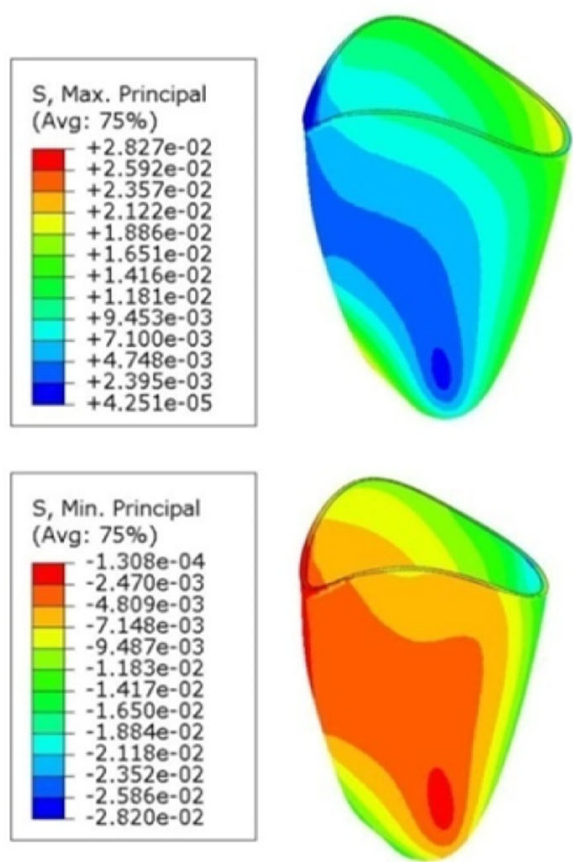

Fig. 7 The nephogram of maximum and minimum principal stresses distribution for the PDL $(M / F=0)$

One limitation of this study is that the FE results are conducted based on one human sample. However, the difference of tooth movement linked to $\mathrm{M} / \mathrm{F}$ ratio between the models with and without bracket, as well as in the buccal-lingual and distal-mesial direction, this method is applicable to any canine. In additional, the results have not compared with clinic experiments. We assume the PDL as a uniform thickness of $0.2 \mathrm{~mm}$ that represents the average thickness. In reality, the PDL's thickness range from 0.15 to $0.38 \mathrm{~mm}$ [30], and it exhibits anisotropic and nonuniform character. Toms SR [31] suggests the stress distributions of the PDL are different for the uniform and nonuniform models. This paper has studied how the bracket and orthodontic force influence $\mathrm{M} / \mathrm{F}$ ratio and the way of tooth movement. Further research is to investigate what will happen to $\mathrm{M} / \mathrm{F}$ ratio and the way of tooth movement when we exert orthodontic force system on different position of tooth crown.

\section{Conclusions}

This study revealed the influences of bracket and force system from different directions on $\mathrm{M} / \mathrm{F}$ ratios, which led to various tooth movements. The M/F ratios of 0 5.6, 6.2, and 10.6 which are generally accepted for uncontrolled tipping, controlled tipping, and translation movements of mandible canine, respectively. These values are smaller for the canine model without bracket, and are larger in buccal-lingual direction than in distal-mesial direction. Therefore, a universal M/F ratio is not recommended. The results of this study have guiding significances for the orthodontist formulate correct therapeutic plan to deal with different situations. Due to the orthodontic forces derived from bracket, wires, and elastic band, it is expected that future tools for orthodontic treatment may be based on the FE results.

\section{Acknowledgements}

The research presented in this paper was supported by School of mechanical Engineering, Southeast University, Nanjing, P.R. China.

\section{Funding}

The authors acknowledge the Fundamental Research Funds for the Central Universities, (Grant: 2242017K3DN02).

\section{Availability of data and materials}

Data sharing not applicable to this article as no datasets were generated or analyzed during the current study.

\section{Authors' contributions}

YY was the main writer of this paper. He proposed the main idea, and completed the simulation, and analyzed the result. WT gave some important suggestions for the performance of this article. All authors read and approved the final manuscript.

\section{Authors' information}

Yu Yang is pursuing his Ph.D. degree in School of Mechanical Engineering, Southeast University. His research interests include biomechanics, Orthodontic simulation.

Wencheng Tang was serving as a professor, and a Ph.D. supervisor. He is currently at work in the School of Mechanical Engineering, Southeast 
University, Nanjing, China. His research interests include advance manufacture technology, element finite analysis, and topology optimization.

\section{Competing interests}

The authors declare that they have no competing interests.

\section{Publisher's Note}

Springer Nature remains neutral with regard to jurisdictional claims in published maps and institutional affiliations.

Received: 10 May 2018 Accepted: 19 June 2018

Published online: 04 July 2018

\section{References}

1. R Savignano, RF Viecilli, A Paoli, AV Razionale, S Barone, Nonlinear dependency of tooth movement onforce system directions. Am. J. Orthod. Dentofac. Orthop 149(6), 838-846 (2016)

2. A Geramy, K Tanne, M Moradi, H Golshahi, YF Jalali, Finite element analysis of the convergence of the centres of resistance and rotation in ectreme moment-to-force ratios. Int. Orithod. 14(2), 161-170 (2016)

3. CJ Burstone, in Vistas in Orthodontics, ed. by B S Kraus, A Ripamonti. The biomechanics of tooth movement (Lea and Febinger, Philadelphia, 1962), pp. 197-213

4. CG Provatidis, Numerical estimation of the centres of rotation and resistance in orthodontic tooth movement. Comput. Methods Biomech. Biomed. Engin 2(2), 149-156 (1999)

5. RL Christiansen, CJ Burstone, Centers of rotation within the periodontal space. Am. J. Orthod 55(4), 353-369 (1969)

6. KR Williams, JT Edmundson, Orthodontic tooth movement analyzed by the finite element method. Biomaterials 5(6), 347-351 (1984)

7. K Tanne, HP Bantleon, Stress distribution in the periodontal ligament induced by orthodontic forces. Use of finite-element method. Inf. Orthod. Kieferorthop 21(2), 185-194 (1989)

8. PM Cattaneo, M Dalstra, B Melsen, The finite element method: a tool to study orthodontic tooth movement. J. Dent. Res 84(5), 428-433 (2005)

9. A Kanjanaouthai, K Mahatumarat, P Techalertpaisarn, Effect of the inclination of a maxillary central incisor on periodontal stress finite element analysis. Angle Orthodonist 82(5), 812-819 (2012)

10. Z Liao, J Chen, $\mathbf{W} \mathrm{Li}$, Biomechanical investigation into the role of the periodontal ligament in optimising orthodontic force: a finite element case study. Arch. Oral Biol 66, 98-107 (2016)

11. Y Cai, B He, X Yang, J Yao, Optimization of configuration of attachment in tooth translation with transparent tooth correction by appropriate momentto-force ratios: biomechanical analysis. Biomed. Mater Eng 26(s1), S507S517 (2015)

12. Y Kojima, H Fukui, A finite element simulation of initial movement, orthodontal movement, and the centre of resistance of the maxillary teeth connected with an archwire. Eur. J. Orthod 36(3), 255-261 (2014)

13. A Merdji, R Mootanah, BAB Bouiadjra, L Aminallah, Stress analysis in single molar tooth. Mater. Sci. Eng 33(2), 691-698 (2013)

14. MR Matson, HR Lewgoy, DA Barros Filho, Finite element analysis of stress distribution in intact and porcelain veneer restored teeth. Comput. Methods Biomech. Biomed. Engin 15, 795-800 (2012)

15. J Yan, H Xianglong, B Cheng, B Ding, Three-dimensional FEM analysis of stress distribution in dynamic maxillary cnaine movement. Chin. Sci. Bull. BiomedicaEng 58(20), 2454-2459 (2013)

16. R Aversa, D Apicella, L Perillo, R Sorrentino, F Zarone, M Ferrari, A Apicella, Non-linear elastic three-dimensional finite element analysis on the effect of endo-crown material rigidity on alveolar bone remodeling process. Dent. Mater 25, 678-690 (2009)

17. IZ Oskui, A Hashemi, Dynamic tensile properties of bovine periodontal ligament: a nonlinear viscoelastic model. J. Biomech 49(5), 756-764 (2016)

18. IZ Oskui, A Hashemi, H Jafarzadeh, Biomechanical behavior of bovine periodontal ligament: experimental tests and constitutive model. J. Mech. Behav. Biomed. Mater 62, 599-606 (2016)

19. M Bergomi, J Cugnoni, M Galli, J Botsis, UC Belser, HW Wiskott, Hydromechanical coupling in the periodontal ligament: a porohyperelastic finite element model. J. Biomechem 44(1), 34-38 (2011)

20. W Li, MV Swain, Q Li, GP Steven, Towards automated 3D finite element modeling of direct fiber reinforced composite dental bridge. J Biomed Mater Res Part B 74(1), 520-528 (2005)
21. HH Ammar, P Ngan, RJ Crout, VH Mucino, OM Mukdadi, Three-dimensional modeling and finite element analysis in treatment planning for orthodontic tooth movement. Am. J. Orthod. Dentofac. Orthop 139(1), 59-71 (2011)

22. YQ Cai, XX Yang, BW He, J Yao, Numerical analysis of tooth movement in different plans of transparent tooth correction therapies. Technol. Health. Care 23, 299-305 (2015)

23. YQ Cai, XX Yang, BW He, Influence of friction in transparent tooth correction treatment: finite element method. J. Mech. Med. Biol 15, 1550052 (2015)

24. Z Wei, X Yu, X Xu, X Chen, Experiment and hydro-mechanical coupling simulation study on the human periodontal ligament. J. Mech. Med. Biol 15(04), 749-759 (2015)

25. WR Proffit, HW Fields, in Contemporary orthodontics, 3rd edn., ed. by P Rudolph. The biological basis of orthodontic therapy (Mosby, St Louis, 2000), pp. 296-325

26. ME Geiger, BG Lapatki, Locating the center of resistance in individual teeth via two- and three-dimensional radiographic data. J. Orofac. Orthop 75(2), 96-106 (2014)

27. T Kondo, H Hotokezaka, R Hamanaka, Types of tooth movement, bodily or tipping, do not affect the displacement of the tooth's center ofresistance but do affect the alveolar bone resorption. Angle Orthod 87(4), 563-569 (2017)

28. T Ouejiaraphant, B Samruajbenjakun, E Chaichanasiri, Determination of the centre of resistance during en masse retraction combined with corticotomy: finite element analysis. J. Orthod. 45(1), 11-15 (2018)

29. PM Cattaneo, M Dalstra, B Melsen, Strains in periodontal ligament and alveolar bone associated with orthodontic tooth movement analyzed by finite element. Orthod. Craniofac. Res 12(2), 120-128 (2009)

30. S Chandra, S Chandra, M Chandra, N Chandra, Textbook of dental and oral histology with embryology (Jaypee Brothers Medical Publishers (P) Ltd., New Delhi, 2007)

31. SR Toms, JE Lemons, AA Bartolucci, AW Eberhardt, Nonlinear stress-strain behavior of periodontal ligament under orthodontic loading. Am. J. Orthod. Dentofacial. Orthop 122, 174-179 (2002)

\section{Submit your manuscript to a SpringerOpen ${ }^{\circ}$ journal and benefit from:}

- Convenient online submission

- Rigorous peer review

- Open access: articles freely available online

- High visibility within the field

Retaining the copyright to your article

Submit your next manuscript at $>$ springeropen.com 